\title{
Further Observations on Changes in the Phage-Typing Pattern of Phage Type 80/81 Staphylococci
}

\author{
By PHYLLIS M. ROUNTREE \\ Fairfax Institute of Pathology, Royal Prince Alfred Hospital, Sydney, \\ New South Wales, Australia \\ AND ELIZABETH H. ASHESHOV \\ Staphylococcus Reference Laboratory, Public Health Laboratory Service, \\ Colindale, London, N.W. 9
}

(Received 17 February 1961)

\section{SUMMARY}

Two distinct temperate phages (594a, 594n) lysogenizing phage type 80/81 staphylococci and producing gains in sensitivity to typing phages 52 and $52 \mathrm{~A}$ were found in lysogenic clones of a strain of staphylococcus of phage type 52/52A/80/81. When either of these phages infected type 80/81 cocci a new phage (594b) appeared in the lysates. This phage was unable to replicate in type $80 / 81$ cocci but could grow in them when they had been lysogenized with phage 594a or $594 \mathrm{n}$. The source of phage 594b was found to be recombination of phage 594a or 594n with a prophage present in cocci of phage type 80/81. This prophage was completely defective and could be demonstrated only by recombination. The gains in phage sensitivity that follow lysogenization of type 80/81 strains with the so-called 'converting' phages can be most satisfactorily explained on the basis of prophage substitution.

\section{INTRODUCTION}

Asheshov \& Rippon (1959) and Rountree (1959) described changes in the phagetyping pattern of staphylococci of phage type $80 / 81$ occurring after lysogenization with certain temperate phages, which were called 'converting' phages for want of a better name. The change of particular interest was that by which the lysogenized cocci became sensitive to the typing phages 52 and $52 \mathrm{~A}$ so that their typing pattern became 52/52A/80/81. The mechanism of this change remained obscure. By replica plating Rountree demonstrated the presence in old broth cultures of the propagating strain of phage 80 (P.S. 80) of mutants sensitive to phages 52 and $52 \mathrm{~A}$ and she suggested that the converting phages might select such pre-existent mutants, which might be more readily lysogenized than the wild type. On the other hand, Asheshov \& Rippon found no mutants sensitive to phages 52 and 52A in their type 80 strain. Furthermore, when they had converted this strain by lysogenization and then 'cured' it of the converting phage by ultraviolet irradiation, the cured strain remained sensitive to phages 52 and 52A. In addition both the original strain and the cured strain gave identical lytic and lysogenic responses to a converting phage. These observations indicated that neither lysogenic conversion nor selection of pre-existing mutants could explain the phenomenon. Prophage substitution 
remained a possibility, but all attempts to demonstrate lysogenicity in cocci of phage type $\mathbf{8 0 / 8 1}$ were unsuccessful. It has now been found, however, that type $80 / 81$ staphylococci contain a completely defective prophage and that the change in the typing pattern after lysogenization with one of the converting phages is due to substitution of this prophage by the converting phage.

\section{METHODS}

Phage type 80/81 staphylococci. Seven staphylococcal strains typing as 80/81 and isolated in a number of different localities were chosen for study. A strain was considered to be phage type $80 / 81$ if it was lysed only by these two phages amongst the 21 phages comprising the basic set of typing phages, both when typing was carried out with the phages at the routine test dilution (RTD) and with the phages at 1000 times greater concentration. The seven type 80/81 strains comprised the following: P.S. 80, the propagating strain for phage 80, isolated in Sydney in 1953. A streptomycin-resistant mutant of P.S. 80 was used in some experiments. It will be referred to as P.S. $80^{\mathrm{sr}} ;$ P.S. 81, the propagating strain for phage 81 , isolated in Canada in 1956 (Bynoe, Elder \& Comtois, 1956); U9, isolated in Philadelphia, Pa., U.S.A. and received from Dr J. Baldwin, Columbus, Ohio, in 1956; 8428, isolated in Oamaru, New Zealand in 1954; 1406/1, obtained from Miss K. Key of the Commonwealth Serum Laboratories, Melbourne, in 1957; 590, isolated in Sydney in 1958; 6346, isolated in England in 1958.

Phage type 52/52A/80/81 staphylococci. Four strains of this phage type were used. They were as follows: strain 594, isolated from the same patient as strain 590 and presumed to have been converted from strain 590 in vivo. Lysogenic clones of this strain were used as the source of two converting phages and are described in detail below; strain $6346 \mathrm{DL}$, derived from strain 6346 which was lysogenized with a converting phage, 72871, and subsequently cured (Asheshov \& Rippon, 1959). This strain is believed to be completely non-lysogenic; strain 80 la, a mutant of P.S. 80 isolated by Rountree by replica plating from an old broth culture of P.S. 80 which differed from the parent culture in being sensitive to the two typing phages 52 and 52A (Rountree, 1959); strain 1406/2, presumed to have been derived from 1406/1 (type 80/81) during mouse passage and differing from the parent culture in being type $52 / 52 \mathrm{~A} / 80 / 81$.

Preparation of phage stocks. Phage stocks were prepared either by ultraviolet (u.v.) induction of singly lysogenic strains or by propagation on a non-lysogenic strain in order to avoid contamination with phages carried by lysogenic strains. The method of u.v. induction was essentially that of Gorrill \& Gray (1956). The cocci were grown with aeration in glucose veal broth for $2 \mathrm{hr}$., centrifuged and resuspended in phosphate buffer $\mathrm{pH} 7 \cdot \mathbf{0}$. They were irradiated with a Westinghouse 'Sterilamp' for $50 \mathrm{sec}$. at a distance of $38 \mathrm{~cm}$., the suspension being agitated in a Petri dish during exposure. The irradiated cocci were inoculated into glucose veal broth at $5 \times 10^{7} \mathrm{cocci} / \mathrm{ml}$. and incubated in a water bath at $37^{\circ}$. After $60 \mathrm{~min}$. they were transferred to the bench where clearing of the culture took place within 2-5 hr. of irradiation. Such stocks had titres of the order of $1-5 \times 10^{9}$ infective particles $/ \mathrm{ml}$. In other cases phage stocks were prepared by propagating the phage on the nonlysogenic strain, $6346 \mathrm{DL}$, by the method of Swanstrom \& Adams (1951).

Counting phages. Agar plates were flooded with a young broth culture of the 
appropriate indicator strain of staphylococcus, the excess broth pipetted off and the plates allowed to dry before the phage dilutions in 0.01 or $0.02 \mathrm{ml}$. volumes were deposited on their surface. Three or four replicates were plated from each dilution and were not spread over the surface of the plate.

Lysogenization. This was carried out on agar as previously described (Rountree, 1959). All phages used for lysogenization were made by u.v. induction, filtered through Gradocol membranes of $0.8 \mu$ A.P.D. and tested for sterility before use. Some strains were lysogenized in broth, a quantity of phage theoretically sufficient to infect all cocci being added to young aerated cultures.

The lysogenized strains are described in the usual way, e.g. P.S. 80 (594a) or, more simply P.S. 80(a) is P.S. 80 lysogenized by the phage derived from strain 594A.

\section{RESULTS}

\section{Phages isolated from strain 594}

Staphylococcus strain 594, of phage type 52/52A/80/81, showed numerous plaques when grown on a phage-typing plate shortly after its isolation, and the original broth culture showed evidence of spontaneous clearing after a day at room temperature. It was plated on agar on two occasions and a number of entire single colonies picked after incubation. These clones, lettered $A$ to $R$, were all of phage type 52/52A/80/81. All of the clones were lysogenic but three different phages were identified in the various clones. The behaviour of these three phages and of cocci lysogenized by them were valuable in demonstrating the mechanism of the conversion of type $80 / 81$ strains to type 52/52A/80/81.

Phage 594a and 594n were converting phages present in the prophage state in clones 594A and 594N, respectively. Both belonged to serological group A and were inducible by u.v. radiation and by superinfection with the other phage; there was, however, no cross-immunity between them.

The third phage, 594b, was found in a single lysogenic clone, 594B. It also belonged to serological group $\mathbf{A}$ and was inducible by $u . v$. radiation and by super infection with phage $594 \mathrm{a}$ or $594 \mathrm{n}$. Cocci of P.S. 80 were immune to it but such cocci became sensitive to this phage after they had been lysogenized by either phage 594a or 594n and could then be used as an indicator for the phage. Clone 594G, doubly lysogenic for 594a and 594n could also be used as an indicator for phage 594b but was less sensitive than $594 \mathrm{~A}$ or $594 \mathrm{~N}$. For the sake of brevity these three phages will be referred to subsequently as phage $a, n$, and $b$.

Table 1 summarizes the reactions of clones isolated from strain 594. Several representatives of each clonal type were identified. The $n$ prophage in clone $\mathbf{F}$ was partly defective since no $n$ phage was produced spontaneously and only a small amount when the clone was induced by u.v. radiation. The host range of the three phages was determined against a set of test strains of various phage types. Only minor differences were detected. The main difference between the three phages therefore lay in their failure to show cross-immunity and in the failure of phage $b$ to lyse strains of phage type $80 / 81$.

\section{Origin of phage $b$}

The origin of phage $b$ and of clone $594 \mathrm{~B}$ was examined. The working hypothesis that strain 594 was derived from a type 80/81 strain, 590, that had been infected 
in vivo with the converting phages, $a$ or $n$, did not explain the occurrence of phage $b$ and clone 594B, since type $80 / 81$ cocci (either P.S. 80 or 590) were immune to the phage. It seemed possible that phage $b$ represented a mutant of phages $a$ or $n$ sufficiently virulent to overcome the immunity of strains lysogenic for phages $a$ or $n$, and which, at the same time, had mutated in such a way that it was no longer able to lyse cocci of P.S. 80. However, high-titre phage preparations made by u.v. induction of strains 594A and P.S. 80 $(a)$ and strains 594N and P.S. 80 $(n)$ showed no trace of phage $b$ when tested on their homologous strains. That phage $b$ arose as a mutant of phage $a$ or $n$ seemed unlikely. Another possibility was that phage $b$ represented a hybrid phage resulting from recombination between phage $a$ and phage $n$. Once again, however, high-titre preparations of phage $a$ propagated on strain 594N and of phage $n$ propagated on strain 594A failed to show the presence of phage $b$. When, however, either phage $a$ or $n$ was propagated on P.S. 80 or strain 590, phage $b$ was regularly recovered in the lysates. It seemed, therefore, that phage $b$ might have its origin in a hitherto undetected prophage in P.S. 80 or else be a recombinant of such a hypothetical prophage with the phage $a$ or $n$.

Table 1. Reactions of clones isolated from staphylococcus strain 594

\begin{tabular}{|c|c|c|c|c|c|c|c|c|c|c|}
\hline \multirow{2}{*}{$\begin{array}{c}\text { Clone } \\
\text { no. }\end{array}$} & \multicolumn{3}{|c|}{ Sensitivity to phages } & \multicolumn{4}{|c|}{ Lysogenicity for } & \multirow{2}{*}{\multicolumn{3}{|c|}{$\begin{array}{l}\text { Prophage } \\
\text { state of } \\
\text { clone }\end{array}$}} \\
\hline & $\vec{a}$ & $b$ & $\vec{n}$ & PS 80 & PS $80(a)$ & $\operatorname{PS~80}(n)$ & 594G & & & \\
\hline 594,A & - & + & + & + & - & + & - & $a$ & . & . \\
\hline $594 \mathrm{~N}$ & + & + & - & + & + & - & - & . & . & $n$ \\
\hline $594 B$ & + & - & + & - & + & + & + & - & $b$ & . \\
\hline $594 \mathrm{C}$ & - & - & + & + & + & + & + & $a$ & $b$ & . \\
\hline 594F & - & + & - & + & $-*$ & + & - & $a$ & - & $n_{d} *$ \\
\hline 594G & - & + & - & + & + & + & - & $a$ & . & $n$ \\
\hline $594 \mathrm{~K}$ & - & - & - & + & + & + & + & $a$ & $b$ & $n$ \\
\hline
\end{tabular}

\section{Lysogenicity of type 80/81 strains}

Previous attempts to demonstrate lysogenicity in type 80/81 strains had been unsuccessful (Asheshov \& Rippon, 1959; Rountree, 1959) but the appearance of phage $b$ during the growth of phage $a$ and $n$ in these strains indicated that a further search should be made. When supernatant fluids of 2-4 hr. broth cultures of P.S. 80 or 590 were filtered and plated on 594A or 594N no plaques were formed. When, however, the supernatant fluids were tested before filtration, minute fuzzy plaques developed which were difficult to count even with a hand lens. When broth cultures were centrifuged for increasing lengths of time, the number of plaques appearing was found to be directly proportional to the number of unsedimented cocci of P.S. 80 in the inoculum. These results suggested that the appearance of plaques was intimately connected with the number of cocci of P.S. 80 able to develop on the surface of the plate. Furthermore, when the unfiltered supernatant fluid of a streptomycinsensitive clone of P.S. 80 was plated on agar containing $1000 \mu \mathrm{g}$. streptomycin $/ \mathrm{ml}$. inoculated with the streptomycin-resistant strains P.S. $80^{\operatorname{sr}}(a)$ or P.S. $80^{\operatorname{sr}}(n)$, a situation in which the unsedimented P.S. 80 cocci were killed, no plaques were seen. When the supernatant of P.S. $80^{\text {sr }}$ was plated under these same conditions, phage plaques were visible, so that failure to see them in the platings from the strepto- 
mycin-sensitive strains was not due to the phage itself being streptomycin-sensitive. Propagation of the phage which produced the fuzzy plaques yielded preparations identical in serological reactions and host range to phage $b$.

It was concluded that no free phage corresponding to phage $b$ was produced by P.S. 80 cocci and that interaction between the converting phages and the type $80 / 81$ cocci was necessary for its production. This interaction would occur on plates if a few type 80/81 cocci were deposited on a lawn of lysogenic cocci that spontaneously released some phage during incubation and thus infected the type 80/81 cocci. The most satisfactory explanation of these findings was that P.S. 80 cocci contained a completely defective prophage that could be detected only when it formed a recombinant with phage $a$ or phage $n$. This prophage will be termed $80^{\prime}$. We have referred to phage $b$ as a single entity regardless of whether it represents a recombinant between phage $a$ and $80^{\prime}$ or phage $n$ and $80^{\prime}$. We have not yet detected any difference between these two recombinants and for the purpose of this paper no distinction will be made between them. A more detailed study of them is in progress.

\section{Prophage substitution during conversion}

If a defective prophage exists in type $80 / 81$ cocci, it is clear that the changes in typing pattern that result from lysogenization might be explicable on the basis of prophage substitution. Type $\mathbf{8 0 / 8 1}$ cocci are immune to the recombinant phage, $b$, and also show 'blocking' of the typing phages 52 and 52A. After lysogenization with a converting phage, such as phage $a$ or $n$, the cocci are sensitive to phages $b$, 52 and 52A. Thus the $80^{\prime}$ prophage may determine the immunity to all three phages and its removal by lysogenization may lead to increased phage sensitivity of the cocci. Further evidence for the loss of defective prophage by substitution with a converting phage is derived from the fact mentioned above, that propagation of phage $a$ in P.S. 80 $(n)$ and vice versa, failed to reveal the presence of any of the recombinant phage, $b$.

It follows that the spontaneous mutants of type 80/81 strains which are sensitive to phage 52 and $52 \mathrm{~A}$ may have gained this phage sensitivity by spontaneous loss of the $80^{\prime}$ prophage. Two such mutants were available, strains 80 la and 1406/2. Both strains were sensitive to phage $b$. Neither strain yielded a recombinant phage when infected with phage $n$, and neither showed the minute fuzzy plaques when their supernatant fluids were plated on strain 594N. Similarly strain $6346 \mathrm{DL}$, which had been lysogenized with a converting phage and subsequently cured, was found to be fully sensitive to phage $b$ and to be incapable of giving rise to it by recombination. It was concluded that all three strains had lost their defective prophage, the first two spontaneously, the latter by prophage substitution, and that this defective prophage determined the typing pattern of the type 80/81 strains and gave rise to the recombinant phage.

\section{Origin of clone $594 B$}

The origin of clone $594 \mathrm{~B}$, the singly lysogenic colony, containing only the $b$ prophage, remained to be explained. It had been isolated from the original broth culture of strain 594 in which the recombinant phage was being produced. Since type $80 / 81$ cocci are resistant to phage $b$ they cannot be lysogenized by this phage. However, a spontaneous mutant which had lost its $80^{\prime}$ prophage would be sensitive 
and could be lysogenized. Alternatively, the doubly lysogenic colonies containing prophage $a$ and $b$ or $n$ and $b$ might be unstable and lose their $a$ or $n$ prophage.

The reconstruction of clones of various prophage states was attempted. Cocci of strain 590 were infected in broth with phage $a$ or phage $n$ and, after clearing, allowed to become turbid again. The culture was diluted and plated so as to obtain 100-200 colonies/plate after incubation. These master plates were replicated on to a plate flooded with strain P.S. 80 as an indicator of phage $a$ or $n$, and on to either strain $594 \mathrm{~A}$ or $594 \mathrm{~N}$, depending on which phage had been used for infection, as an indicator of phage $b$. By this means, colonies containing prophages $a$ alone, $n$ alone, $a$ and $b$, and $n$ and $b$ were identified and isolated. Some of the colonies containing $a$ and $b$ or $n$ and $b$ were unstable when subcultured in broth and showed plaques when flooded on to agar plates, thus behaving like 'suicide' strains. Some single colonies picked from subcultures of these strains contained only the $b$ prophage. Similarly, although doubly lysogenic colonies were readily obtained when clones $594 \mathrm{~A}$ or $594 \mathrm{~N}$ were treated with phage $b$, such cultures were sometimes unstable on continued subculture and cocci containing the $b$ prophage alone could be isolated from them. The singly lysogenic clone $594 \mathrm{~B}$ could therefore have arisen either by lysogenization of a spontaneous mutant or else by double lysogenization, first with a converting phage and second with the recombinant phage, and the subsequent loss of the 'converting' prophage.

\section{Lysogenicity of other type 80 strains}

All of the seven type 80/81 strains examined have yielded the recombinant phage after infection with phage $n$. Table 2 shows the count of phage $n$ and phage $b$ at the end of one-step growth experiments of phage $n$ in five strains isolated from widely different places. All five strains were examined for the presence of free phage but none could be found and it was concluded that all contained the defective prophage. All changed their typing pattern to 52/52A/80/81 after lysogenization with phage $n$.

In addition, 85 strains which had been sent to the Staphylococcus Reference Laboratory in London for routine phage typing and which were phage type 80/81 when tested with the typing phages at the routine test dilution (RTD) were examined for carriage of the defective phage in the following way. The supernatant fluids of $24 \mathrm{hr}$. broth cultures of the strains were spotted on to plates flooded separately with strains $594 \mathrm{~A}, 594 \mathrm{~N}, 594 \mathrm{~B}$, and the non-lysogenic strain $6346 \mathrm{DL}$. The appearance of small fuzzy plaques on the first two strains and the absence of any reaction of the latter two strains was considered to be fairly good evidence of the carriage of the defective prophage $80^{\prime}$. Of the 85 strains 68 gave the expected result; 10 of the 68 were tested with the typing phages at 1000 RTD and all 10 were type $80 / 81$. The supernatant fluids of the remaining 17 strains showed strong lytic reactions on all four indicator strains. On typing these strains at 1000 RTD all 17 were found to be type 52/52A/80/81, i.e. not typical 'phage type $80 / 81$ ' strains. These results indicated that the carriage of the defective prophage $80^{\prime}$ was characteristic of all typical type $80 / 81$ strains.

\section{Frequency of recombination}

One-step growth experiments were run to determine the rate at which phage $a$ or phage $n$ recombined with phage $80^{\prime}$ to form the recombinant phage $b$. A log-phase broth culture of P.S. 80 was spun down, washed once in phage adsorption medium 
Table 2. Production of phage $594 b$ by staphylococcal strains of type 80 infected with phage 594n; counts made at the end of one step growth curves

$\begin{array}{ccc}\text { Strain no. } & \begin{array}{c}\text { Phage 594n } \\ \text { (particles } / \mathrm{ml} \text {.) }\end{array} & \begin{array}{c}\text { Phage 594b } \\ \text { (particles } / \mathrm{ml} \text {.) }\end{array} \\ \text { PS 80 } & 1 \cdot 7 \times 10^{9} & 5 \cdot 9 \times 10^{6} \\ \text { PS 81 } & 3 \cdot 9 \times 10^{9} & 4 \cdot 2 \times 10^{6} \\ \text { U9 } & 2 \times 10^{9} & 7 \times 10^{6} \\ 8428 & 2 \cdot 6 \times 10^{9} & 1 \cdot 1 \times 10^{7} \\ 1406 / 1 & 6 \cdot 4 \times 10^{9} & 2 \cdot 7 \times 10^{6}\end{array}$

(PAM; Hershey \& Chase, 1952) and finally resuspended in PAM to a concentration of $1-2 \times 10^{8}$ viable units $/ \mathrm{ml}$. Sufficient phage $a$ or $n$ was added to infect about $40-50 \%$ of the cocci in $15 \mathrm{~min}$. at $37^{\circ}$. At the end of this time the adsorption mixture was centrifuged, washed once in PAM and finally resuspended in nutrient broth warmed to $37^{\circ}$, the time of resuspension being taken as $0 \mathrm{hr}$. Tenfold dilutions were made in warm nutrient broth to a point where $0.1 \mathrm{ml}$. contained about $1 \times 10^{2}$ infected cocci $/ \mathrm{ml}$. The dilution tubes were held at $37^{\circ}$ throughout the experiment and at intervals samples were removed from the appropriate dilutions and titrated for phage $a$ (or $n$ ) and for the recombinant phage. Titrations for the recombinant phage were made on agar containing $1000 \mu \mathrm{g}$. streptomycin $/ \mathrm{ml}$. The streptomycinresistant indicator strain $80^{\mathrm{sr}}(a)$ was used to titrate the recombinant phage where phage $a$ was the infecting phage and strain $80^{\operatorname{sr}}(n)$ where phage $n$ was the infecting phage. In the presence of streptomycin, uninfected cocci of P.S. 80 which are deposited on the plate are unable to grow and no recombination can take place on the plate. Infected cocci in which recombination has taken place at the time of plating will probably produce a plaque, provided that at least one recombinant particle is already mature or matures within 2-3 min. of plating; cyanide-lysis experiments had shown that streptomycin at the concentration used required this length of time to exert its inhibitory effect. As a control on the streptomycin effect, titrations for phage $a$ (or $n$ ) were made on nutrient agar and on streptomycin agar plates both flooded with strain $80^{\text {sr }}$.

The results of a typical experiment are shown in Fig. 1. In this experiment phage $a$ which was used to infect P.S. 80 had a latent period of about $35 \mathrm{~min}$. and an average burst size of 20 particles of phage $a /$ infected coccus. The actual burst size may be smaller since, staphylococci having a tendency to clump, the actual number of infected cocci during the latent period may be higher than the number recorded. Plaques of phage $a$ on streptomycin agar began to appear at $5 \mathrm{~min}$. and increased logarithmically up to $35 \mathrm{~min}$., when the count equalled that on nutrient agar. This was interpreted to mean that mature particles of phage $a$ appeared at $5 \mathrm{~min}$. when some $3 \cdot 4 \%$ of infected cocci contained at least one mature phage particle. There was a delay in the appearance of mature recombinant particles until $20 \mathrm{~min}$. At this time about $50 \%$ of infected cocci contained mature $a$ particles while only $0.0002 \%$ contained a recombinant particle. The proportion of infected cocci containing recombinant particles increased rapidly until at $35 \mathrm{~min}$. the proportion was about $0.07 \%$.

It was not clear from the results of a number of experiments whether or not the cocci that released the recombinant phage released more than one recombinant 
particle. In most experiments the count of phage $b$ continued to increase during the rise period to a final concentration approximately $2-3$ times the count at the beginning of the rise period. This might represent a true burst size for the recombinant or it might be the result of late-lysing cocci contributing to the count of recombinant particles. When the infected cocci were lysed with cyanide during the latent period, curves such as that for phage $n$ shown in Fig. 2 were obtained. Replication of phage $n$ was complete at $50 \mathrm{~min}$. but the release of the phage was not complete until $60 \mathrm{~min}$. During this last $10 \mathrm{~min}$. period the amount of phage $b$ increased from $7 \cdot 1 \times 10^{5} / \mathrm{ml}$. to $9 \cdot 5 \times 10^{5} / \mathrm{ml}$. These results do not exclude the possibility of the replication of the recombinant in individual cocci. They do, however, suggest that the initial step of recombination occurs later in the latent period than the initial step of replication of the infecting phage which is in keeping with current knowledge of the recombination process in coli phages (Hershey, 1958).

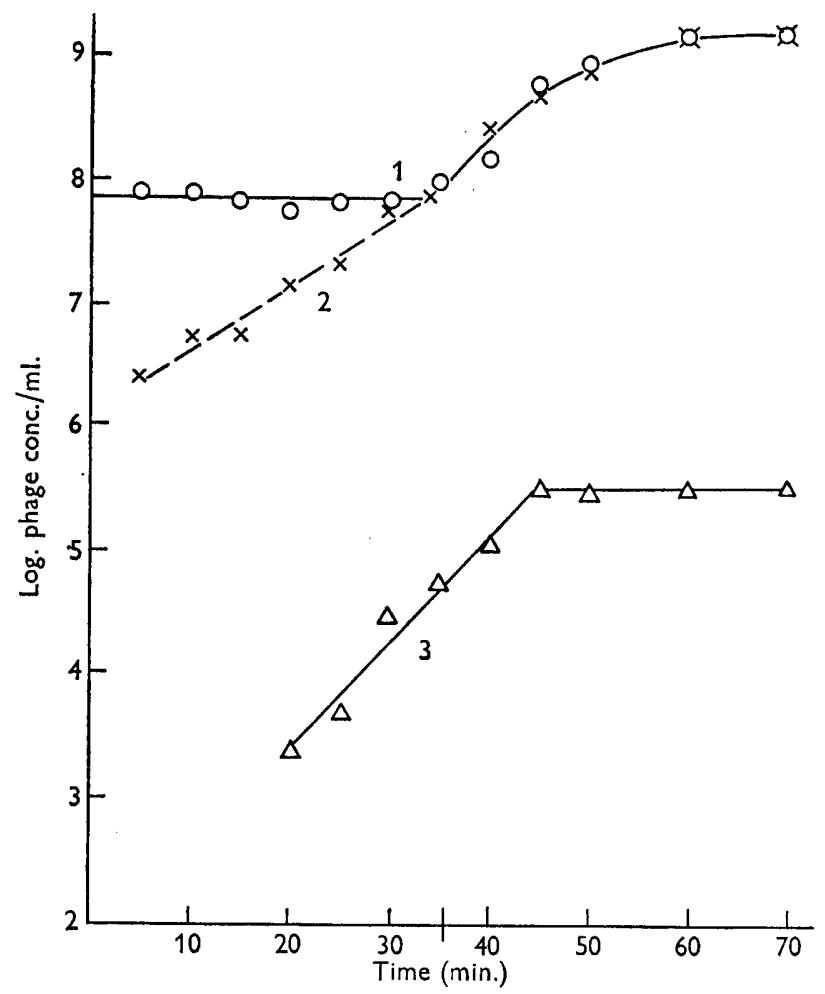

Fig. 1. One step growth curve of phage $a$ in staphylococcus PS 80. Titrations for phage $a$ were made on strain $80^{\text {sr }}$ on plain agar (curve 1 ) and on streptomycin agar $(1000 \mu \mathrm{g} . / \mathrm{ml}$.) (curve 2). Titrations for the recombinant phage $b$ were made on strain $80^{\mathrm{sr}}(a)$ on streptomycin agar (curve 3).

The rate of recombination was calculated as the ratio of the number of infected cocci releasing the recombinant to the total number of infected cocci. However, without knowing whether the increase in the number of recombinant particles during the rise period represented an actual burst size or simply the contribution of late-lysing cocci it is impossible to know how many infected cocci actually released 
at least one recombinant particle. If it be assumed that the recombinant replicates in those cocci that produce it and that the increase represents a true burst, the ratio would be best calculated on the number of cocci releasing any recombinant phage at the end of the latent period. If, on the other hand, the increase in the recombinant during the rise period be due entirely to the contribution of late lysing cocci, and no replication of the recombinant takes place in any coccus that releases it, then the ratio should be calculated on the total amount of the recombinant released at the end of the rise period.

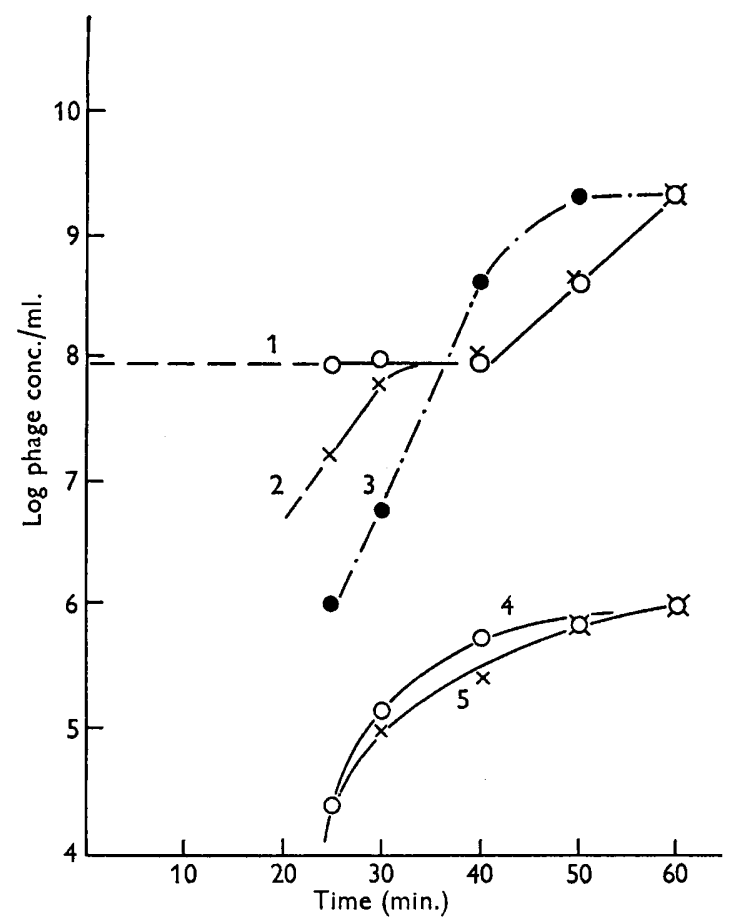

Fig. 2. Cyanide lysis experiment of staphylococcus PS 80 infected with phage $n$. Infected cocci were diluted into broth containing $0 \cdot 02 \mathrm{M}$ sodium cyanide after $25,30,40$ and $50 \mathrm{~min}$. These dilutions were held at $4^{\circ}$ for $30 \mathrm{~min}$. then at $37^{\circ}$ for $60 \mathrm{~min}$. before titrating for phage $n$ on strain $80^{\mathrm{sr}}$ and for the recombinant phage $b$ on $80^{\mathrm{sr}}(n)$. Curve $1=$ phage $n$ titrated on plain agar. Curve $2=$ phage $n$ titrated on agar containing $10 \mu \mathrm{g}$. streptomycin $/ \mathrm{ml}$. Curve $3=$ phage $n$ released by cyanide-lysed cocci at the time indicated. Curve $4=$ phage $b$ titrated on streptomycin $(10 \mu \mathrm{g} . / \mathrm{ml}$.) agar. Curve $5=$ phage $b$ from cocci prematurely lysed by cyanide at the times indicated.

The figures obtained differed in different experiments but there was little difference regardless of whether phage $a$ or phage $n$ was used as the infecting phage. When calculations were based on the count of the recombinant at the end of the latent period (assuming a small burst size) the figures varied between 1/100 and 1/1000 with an average of about $1 / 600$. When calculations were based on the final count of the recombinant (assuming no replication of the recombinant) the figures varied between $1 / 30$ and 1/250 with an average of about $1 / 150$. 


\section{DISCUSSION}

The experimental results described in this paper are compatible with the hypothesis that the 'conversion' of type 80/81 staphylococci to type 52/52A/80/81 after lysogenization is due to prophage substitution. A similar example of prophage substitution in staphylococci was described by Gorrill (1957) who showed that a prophage carried by the propagating strain of typing phage $\mathbf{7 6}$ was displaced when the strain was lysogenized with phage 76. There are several similarities between the system studied by Gorrill and that investigated in the present paper. These include the instability of some of the doubly-lysogenic colonies and the gain in phage sensitivity following lysogenization. The main difference between the present system and that of Gorrill is the complete defectiveness of the $\mathbf{8 0}^{\prime}$ prophage. The prophage state in the type 80/81 cocci resembles that described by Cohen (1959) for the prophage of Escherichia coli $\mathrm{B}$, which is completely defective and recognizable only by its ability to enter into recombination with phage $\mathbf{P} 2$.

The present results throw some light on to the relationship of the prophages in type $80 / 81$ cocci. The presence of the $80^{\prime}$ prophage causes a 'blocking' of the sensitivity of the cocci to infection with phages 52 and 52A. It may be postulated that this blocking is due to a steric interference by the $\mathbf{8 0}^{\prime}$ prophage which makes loci for phage 52 and $52 \mathrm{~A}$ inaccessible to these phages. When the $80^{\prime}$ prophage is displaced by the converting phages or by spontaneous loss, this steric interference disappears. The recombinant phage $b$ can lysogenize converted type $80 / 81$ cocci from which the $80^{\prime}$ prophage has disappeared and this does not cause blocking of phage 52 and 52A. In other words, interference with phages 52 and $52 \mathrm{~A}$ is a characteristic of $80^{\prime}$ prophage but not of its recombinant. If, as Jacob \& Wollman's work (1959) implies, attachment of the prophage to the bacterial chromosome may involve overlapping by the prophage of some genetic loci, then it may be postulated that the prophages $a, n$ and $b$ overlap fewer sites than does the $80^{\prime}$ prophage.

The phages $a, n$ and $b$ are closely related. Work in progress shows that infection with any of them will induce the others when they are present as prophages. It was considered that phage $a$ might be a 'dismune' (Bertani, 1958) mutant of phage $n$ or vice versa. However, no evidence for such a mutation has been found. It must therefore be concluded that the original type 80/81 strain in the patient from whom the 594 clones were isolated was infected with two converting phages in the respiratory tract.

Cocci of type $80 / 81$ infected with a converting phage in broth culture behave as suicide cultures. They survive the attack of the phage by becoming lysogenized with it; this, however, renders them sensitive to the recombinant phage which has been produced in a proportion of the infected cocci and on plating they will show plaques due to this phage. If such broth cultures are continued they may show a second clearing. The cocci surviving this second attack will be doubly lysogenic. Strains Bundaberg, 308, 313 and 315 which had been previously used as sources of converting phages (Rountree, 1959) were examined and found to be resistant to phages $a$ and $b$ but sensitive to phage $n$ and it was concluded they were doubly lysogenic. On the other hand, strain 9684, which was the source of a converting phage identical with that of Bundaberg, had shown free phage on its original plate 
and then been picked four times from single colonies; it contained only prophage $a$ and had evidently lost the recombinant phage.

The type $80 / 81$ strains used in this study were selected as being representative of isolations made in widely separated parts of the world. The fact that all behaved similarly on lysogenization supports the view that they are closely related, if not identical. This similarity is not, however, proof of a single common origin of all these strains.

Some interesting problems are posed by the appearance of the recombinant phage following infection with the converting phages. So far as we are aware, there is no information on phage crosses and recombination in staphylococcal phages, since experimental systems with suitable genetic markers are at present lacking. On analogy, however, with the coli phages, recombination in the $a$ or $n$ phages would imply that the $80^{\prime}$ prophage is able to enter the mating pool of the infecting phages. The late maturation of the recombinant particles compared with the infecting particles suggests either that this entry occurs at a relatively late stage in the latent period or that, entry having occurred, there is a random chance of the $80^{\prime}$ prophage forming a recombinant. Further, there is no definite evidence for or against further replication of the recombinant. Entry into the mating pool also implies that, after infection with the converting phage, there is an alteration in the physical state of the $80^{\prime}$ prophage leading to its detachment from its site on the bacterial chromosome. Whether this happens in every type $80 / 81$ coccus infected with a converting phage is unknown but it certainly occurs in all cocci giving a lysogenic response to infection, since all of these lose their $80^{\prime}$ prophage.

The term 'converting' phage has been used to describe the phages concerned in this phenomenon. The term is inaccurate when applied to phages $a$ or $n$, since the change in the typing pattern of strains of type $80 / 81$ which results from lysogenization with either of these phages is not due to the presence of the phage per se but simply to the loss of the defective phage $80^{\prime}$. All the evidence suggests, however, that phage $80^{\prime}$ is a converting phage since its presence in the coccus is essential for maintaining the typing pattern of the strain. Its loss, either spontaneously or by prophage substitution, caused an immediate change in the typing pattern. A closer analysis is required of the characteristics of individual recombinants following infection with different phages in order to find out what variations, if any, there are in the genetic contributions made to the recombinants by the infecting phages.

This work was supported in part by a grant from the Australian National Health and Medical Research Council.

\section{REFERENCES}

Asheshov, E. H. \& Rippon, J. E. (1959). Changes in typing pattern of phage-type 80 staphylococci. J. gen. Microbiol. 20, 634.

Bertani, G. (1958). Lysogeny. Advanc. Virus Res. 5, 151.

Bynoe, E. T., Elder, R. H. \& Comtors, R. D. (1956). Phage-typing and antibiotic resistance of staphylococci isolated in a general hospital. Canad. J. Microbiol. 2, 346.

Coren, D. (1959). A variant of phage P2 originating in Escherichia coli strain B. Virology, $7,112$.

GorRILl, R. H. (1957). Studies on lysogeny in staphylococci. J. gen. Microbiol. 17, 254.

Gorrill, R. H. \& Gray, R. A. (1956). 'The induction of bacteriophage in staphylococci. J. gen. Microbiol. 14, 167. 
Hershey, A. D. (1958). The production of recombinants in phage crosses. Cold Spr. Harb. Symp. quant. Biol. 23, 19.

Hershey, A. D. \& Chase, M. (1952). Independent functions of viral protein and nucleic acid in growth of bacteriophage. J. gen. Physiol. 36, 39.

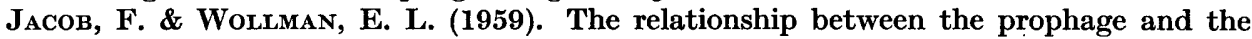
bacterial chromosome in lysogenic bacteria. Recent Progress in Microbiology. Stockholm: Almquist and Wiksell.

Rountree, P. M. (1959). Changes in the phage-typing patterns of staphylococci following lysogenization. J. gen. Microbiol. 20, 620.

Swanstrom, M. \& Adams, M. H. (1951). Agar layer method for production of high titre phage stocks. Proc. Soc. exp. Biol., N.Y. 78, 372. 